\title{
Special radiocarbon section
}

Two 'radiocarbon revolutions' have been announced, a first when Libby's absolute-dating method was made to work in the 1950 s, a second when first-generation calibration upturned some European chronology in the 1960s. Declaring a third would make at least two radiocarbon revolutions too many (and all inside 40 years). So let us call what is happening at present a reformation, and remember how radical and disconcerting a reformation can be.

This special section tries to show some of what that reformation is doing. It has four elements.

The first is small-sample dating, either with miniature conventional counters or by the AMS system, which examines directly the ratio of $14 \mathrm{C}$ to $12 \mathrm{C}$ atoms rather than waiting for $14 \mathrm{C}$ to decay naturally. These methods reduce the sample size so much that many things can now be reliably dated which could not be touched before.

The second is the new calibration curves, not yet definitive for all periods but likely to endure without gross modification for perhaps the next 10 years. In the early days it was unclear whether the curve would be wiggly or not far from a straight line. Now we know the curve is truly wiggly, and this has important consequences: it may be of slight use, for example, to take tiny charcoal samples for AMS dating from a late iron-age ditch, so as to plot its silting, because the re-entrant curve in the period immediately before 1 BC makes calibration insensitive there.

The third element is statistical: the correct evaluation of a set of radiocarbon measurements requires exacting mathematical treatment. It is not sufficient to think of each determination as a simple approximation to the central quoted date, or even to calibrate each date by itself. Precise mathematical methods are needed to extract the true information a set of dates conveys.

The fourth and final element is a new and proper concern with just what a radiocarbon measurement measures. What stochastic and laboratory errors may there be? Which are allowed for in the quoted standard deviation? How old was the carbon when it was incorporated into the archaeological deposit? Is the deposit stratigraphically secure? is it securely associated with the archaeological event or episode to be dated? These make some radiocarbon determinations more equal than others, and render many worthless.

This section has six articles:

Gordon Pearson sets out how to cope with calibration - and how best to benefit from it.

E.T. Hall applies AMS to a single artefact. His artefact is rather recent, so calibration is not critical, and there is direct dendrochronology for a second opinion.

Alan Saville, John Gowlett \& Robert Hedges take a suite of AMS samples from a single site, apply calibration, and can make some startling inferences for the wider pattern of the British Neolithic.

Fekri Hassan \& Steven Robinson take the available measurements for a single regional cultural sequence, that of ancient Egypt, discard the many determinations that are unusable, and calibrate the rest to make for the first time - a workable chronology for Egypt and its region that is independent of the documentary record.

Barbara Ottaway summarizes what we can do and cannot yet do in working with calibration.

Robin Dennell reviews the Oxford AMS unit's experience so far, underlining the achievement, and pointing to the problems that still press.

Making the reformation work is not going to be easy. The mathematics are beyond most of us. There has to be more care in just what is dated and why. A ragbag of measurements, taken often singly by different laboratories at different times on different materials from different contexts of different stratigraphic security, has been the norm for arguments over episodes like the Wessex culture - arguments which, it is now clear, are chronologically pretty meaningless.

New archaeological problems are arising. The dating of single carbonized grains, as Robin Dennell underlines, shows that they can move many centimetres vertically between strata; so how much confidence can there be now in the proofs of early cultivation provided by a few grains found in a 'sealed' early deposit?

CHRISTOPHER CHIPPINDALE 